\title{
Effect of Cleistanthins A and B on the Isolated Aorta of Guinea Pig
}

\author{
Firoj Kumar Sahoo, Chandrashekaran Girish, Ramaswamy Raveendran \\ Department of Pharmacology, Jawaharlal Institute of Postgraduate Medical Education and Research, Puducherry, INDIA.
}

\begin{abstract}
Objectives: Cleistanthins $A$ and $B$ are the active compounds isolated from Cleistanthus collinus leaves. The hypotensive activity of both cleistanthins $A$ and $B$ and the leaf extract has been previously demonstrated, but the exact mechanism of action is not clear. So, this study was designed to test the vasorelaxant property of cleistanthins $A$ and $B$ and delineate its mechanism of action. Methods: Variable force transducers were used to record the force of contraction from the aortic rings of 24 male guinea pigs. The effect of cleistanthin $A$, cleistanthin $B$, prazosin and vehicle on the phenylephrine-induced contractions and potassium chloride-induced contractions was demonstrated and compared. Results: Cleistanthin B inhibited the phenylephrine-induced contractions in a dose-dependent manner and demonstrated potency $\left(\mathrm{plC}_{50}=6.8 \pm 1.06\right)$ comparable to prazosin $\left(\mathrm{plC}_{50}=5.7 \pm 0.7\right)$. Cleistanthin $A$ potency could not be calculated. Both cleistanthin A $(4 \mu \mathrm{g})$ and cleistanthin B $(40 \mu \mathrm{g})$ caused a reduction of the maximal response of phenylephrine demonstrating irreversible blockade. They did not affect the potassium chloride-induced
\end{abstract}

contractions. This illustrates that cleistanthins $A$ and $B$ are noncompetitive $\alpha_{1}$ adrenergic blockers and the potency of cleistanthin $B$ is comparable to prazosin. Conclusion: Both cleistanthins $A$ and $B$ have vasodilatory action on guinea pig aorta. The action is through non-competitive $a_{1}$ adrenergic receptor inhibition and calcium channel blockade does not contribute to the inhibition.

Key words: $a_{1}$ adrenergic antagonist, Cleistanthin A, Cleistanthin B, Cleistanthus collinus, Guinea pig, Isolated aortic rings.

\section{Correspondence}

Dr. Chandrashekaran Girish

Department of Pharmacology, Jawaharlal Institute of Postgraduate Medical Education and Research, Puducherry-605006, INDIA.

Email: gcnx@rediffmail.com

DOI: 10.5530/jyp.2021.13.23

\section{INTRODUCTION}

Cleistanthus collinus [Family: Phyllanthaceae] leaves are notorious for being commonly used as a suicidal agent in southern states of India with many case reports describing the same. ${ }^{1-4}$ The multisystem involvement and electrolyte disturbances (specifically hypokalemia) are the most common features of toxicity. ${ }^{5-7}$ Some patients have developed severe hypotension not reversed even with strong vasoactive agents. ${ }^{1,3}$ Supportive management with correction of electrolyte imbalance is the mainstay of treatment as no antidote exists. ${ }^{7}$

Search for the active constituents in Cleistanthus collinus leaves responsible for its toxicity led to the identification, isolation and purification of two dyphylline glycosides, cleistanthins A and B..$^{8-12}$ Most toxic manifestations of Cleistanthus collinus poisoning have been successfully linked to cleistanthins A and B, but what has spurred the interest in these novel compounds is their cytotoxic potential. ${ }^{1,4}$ In vitro and in vivo data suggest promising anticancer, antimicrobial and larvicidal activities as well. ${ }^{13-17}$ However, hypotension caused in patients of Cleistanthus collinus poisoning is concerning. The therapeutic potential of these can be affected by the off-target hypotensive effects. The mechanism of hypotension is not well understood. On the other hand, due to the hypotensive effects, cleistanthins A and B have been tried in animal models of hypertension with success and could also reduce blood pressure in anaesthetized rats. ${ }^{18-20}$ Thus, a detailed study of the mechanism of hypotension is of interest.

Although $\mathrm{H}^{+}$-ATPase blockade is the responsible mechanism for most of the actions of cleistanthins $\mathrm{A}$ and $\mathrm{B}$ and the manifestations of Cleistanthus collinus poisoning, it does not explain the hypotension produced..$^{21-23}$ The ability of cleistanthins $\mathrm{A}$ and $\mathrm{B}$ to relax guinea pig vas deferens, a tissue rich in $\alpha_{1}$ adrenergic receptors, points to $\alpha_{1}$ adrenergic receptor blockade as a possible mechanism. ${ }^{24}$ However, nonspecific calcium channel blockade can also lead to smooth muscle relaxation and has to be ruled out. ${ }^{25-27}$ The nature and potency of this blockade have also not been characterized. The leaf extract of Cleistanthus collinus has been reported to relax the guinea pig aorta, but a similar effect of cleistanthins $\mathrm{A}$ and $\mathrm{B}$ on the aorta is yet to be demonstrated. ${ }^{28}$ Leaf extract of Cleistanthus collinus contains more than 30 compounds and it is difficult to attribute this effect to cleistanthins A and B unless we study their effects on the aorta independently. ${ }^{29}$ Functional tissue experiments with guinea pig aortic rings can address the above issues efficiently. Inhibition of phenylephrine-induced contractions can provide evidence for $\alpha_{1}$ adrenergic antagonism and the absence of inhibition of potassium chloride-induced contractions can be used to rule out calcium channel blockade. ${ }^{25-27}$

Thus, our study was designed to measure the degree of inhibition by cleistanthins $\mathrm{A}$ and $\mathrm{B}$ on phenylephrine-induced guinea pig aortic ring contractions and to compare their potency with prazosin in doing so. Also, an attempt has been made to rule out a possible calcium channel blocking the action of cleistanthins A and B by studying its action on potassium chloride-induced contractions.

\section{MATERIALS AND METHODS}

\section{Test compounds, drugs and solutions}

Cleistanthins A and B were isolated from the acetone extract of Cleistanthus collinus leaves as described by Parasuraman et al. (2009). ${ }^{8}$

This is an open access article distributed under the terms of the Creative Commons Attribution-NonCommercial-ShareAlike 4.0 License, which allows others to remix, tweak, and build upon the work non-commercially, as long as the author is credited and the new creations are licensed under the identical terms. 
Solutions of Cleistanthins A and B were prepared by diluting concentrated solutions of cleistanthins $\mathrm{A}$ and $\mathrm{B}$ in absolute ethanol with distilled water to obtain desired concentrations. Phenylephrine and prazosin pure powder were purchased from Sigma-Aldrich (Missouri, USA). Distilled water was used as the solvent to prepare desired concentrations of phenylephrine and prazosin solutions. Potassium chloride $(\mathrm{KCl})$ and reagents for the Kreb's-Henseleit solution used in the tissue experiment were procured from Merck chemicals (India). Kreb's-Henseleit solution used in the experiment was prepared by adding sodium chloride $(118 \mathrm{mM})$, potassium chloride $(4.7 \mathrm{mM})$, sodium hydrogen carbonate $(25 \mathrm{mM})$, potassium dihydrogen orthophosphate $(1.2 \mathrm{mM})$, dextrose (11.1 mM), magnesium sulfate $(1.2 \mathrm{mM})$ and calcium chloride $(2.5 \mathrm{mM})$ to distilled water.

\section{Study groups and Animals}

Approval for the study was obtained before the study from the Institute Animal Ethics Committee (IAEC), JIPMER, India. Healthy male guinea pigs weighing 300-400 g obtained from the central animal house (JIPMER, India) were used in the study. The animals were kept under standard laboratory conditions, with food and water ad libitum. A total of twenty-four guinea pigs were randomly divided into four groups with six animals in each group. The four groups used are, Group A (cleistanthin A group), Group B (cleistanthin B group), Group C (prazosin group) and Group D (vehicle control group).

\section{Isolated tissue experiment}

The guinea pigs were sacrificed by exsanguination and dissected to harvest the thoracic aorta. The dissected aortic tissue was then placed in a petri dish containing Kreb's-Henseleit buffer solution as the physiological salt solution (PSS). The aortic tissue was then cut carefully into rings measuring 1-2 cm in length. With the help of two L-shaped metal hooks, each ring was placed inside a $40 \mathrm{ml}$ tissue bath containing PSS maintained at $37^{\circ} \mathrm{C}$ and aerated with carbogen $\left(95 \% \mathrm{O}_{2}+5 \% \mathrm{CO}_{2}\right)$ gas. The tension in the rings was measured using variable force transducers (Biopac data acquisition system). A standard resting tension of $2 \mathrm{~g}$ for 2 $\mathrm{h}$ was used to allow full relaxation. The baseline was recorded and the relaxed tissue was subjected to the test protocols. The tissues from each animal in groups A and B were subjected to three protocols (inhibition of phenylephrine-induced contractions, prevention of phenylephrineinduced contractions and inhibition of potassium chloride-induced contractions) while groups $\mathrm{C}$ and $\mathrm{D}$ were subjected to only 2 protocols (inhibition of phenylephrine-induced contractions and prevention of phenylephrine-induced contractions). A fresh aortic ring was used for each protocol.

\section{Inhibition of phenylephrine-induced contractions (curative protocol)}

In this protocol, the ability of the test drugs to relax aortic tissues precontracted with phenylephrine was assessed. Each of the relaxed aortic rings in this protocol was subjected to a phenylephrine concentration of $1.5 \mathrm{M}$ in the tissue bath to induce maximal contraction. After the response had stabilized, increasing doses of the test drugs (cleistanthins $\mathrm{A}$ and $\mathrm{B}$, prazosin or vehicle control based on the group being studied) were added $12 \mathrm{~min}$ apart to relax the fully contracted tissue sequentially. The final concentration in the tissue bath after each such dose was noted. This was repeated till maximal inhibition was achieved or the maximal permissible volume to be added to the tissue bath (5\% of tissue bath volume) was achieved. A dose-response curve was obtained by plotting the percentage response against the log concentration of the test drug. The final concentration of the test drugs in the tissue bath required to inhibit $50 \%$ of the contraction $\left(\mathrm{IC}_{50}\right)$ was calculated and compared.

\section{Prevention of phenylephrine-induced contractions (preventive protocol)}

In this protocol, the graded phenylephrine dose response curves obtained before and after adding the test drug were compared. The graded phenylephrine dose response curves were obtained by adding incremental doses of phenylephrine to the relaxed tissue 6 min apart and plotting the percentage response obtained against the concentration of phenylephrine in the tissue bath after each dose. The tissue was then relaxed completely by frequent washing. Then it was incubated with the test drug (cleistanthin A, cleistanthin B, prazosin or vehicle control based on the group being studied) for $45 \mathrm{~min}$ following which the graded phenylephrine dose response curve was repeated. The percentage response at each concentration before and after adding the test drugs was plotted against the log dose of phenylephrine and compared. The concentration of phenylephrine eliciting $50 \%$ of the maximal response before ( $\mathrm{EC}_{50}$ before the test) and after ( $\mathrm{EC}_{50}$ after test) adding the test drug was noted.

\section{Inhibition of potassium chloride-induced contractions}

The fully relaxed aortic rings were contracted by using potassium chloride instead of phenylephrine. The physiological salt solution in the tissue baths containing the aortic rings was replaced with a physiological salt solution containing a high concentration of potassium chloride $(80 \mathrm{mEq})$ to induce the contractions. The contraction of the tissue was allowed to stabilize before starting the challenge with the test compounds. The response was noted and increasing doses of the test compounds (cleistanthin A or B) were added in the tissue bath at a time interval of $12 \mathrm{~min}$ to achieve desired incremental concentrations of the test compounds in the tissue bath. The response at the end of $12 \mathrm{~min}$ was noted before the next dose. The concentrations of cleistanthins A or B used in this protocol were the same as that used for phenylephrineinduced contractions in the curative protocol. To demonstrate the role of calcium channel blockers in the inhibition of potassium chlorideinduced contractions two aortic rings precontracted with potassium chloride were relaxed with increasing doses of diltiazem, a calcium channel blocker.

\section{Data Analysis}

The responses at each dose were converted to the percentage of maximal response for all protocols and were expressed as mean \pm standard error of the mean. Comparisons between the groups at each dose were made using Two-way repeated-measures ANOVA with Bonferroni's post-hoc test for multiple comparisons. $\mathrm{The} \mathrm{IC}_{50}$ values obtained from the curative protocol were log-transformed to $\mathrm{pIC}_{50}$ (negative logarithm of $\mathrm{IC}_{50}$ ) to attain normal distribution of data. The $\mathrm{pIC}_{50}$ values were expressed as mean \pm standard deviation and were compared between the groups using the Student's t-test. A $p$ value of less than 0.05 was considered significant. GraphPad Prism ver 8.3.1 was used for the analysis of data.

\section{RESULTS}

Cleistanthins A and B and vehicle contracted the guinea pig aortic rings independently in a dose-dependent manner. The dose response curves for the three based on the ethanol content are displayed in Figure 1. The responses produced by all three were inhibited by prazosin.

\section{Inhibition of phenylephrine-induced contractions}

The effect of cleistanthins A and B on the guinea pig aortic tissue precontracted with phenylephrine is demonstrated by Figures $2(\mathrm{a}, \mathrm{b}, \mathrm{c})$. The values plotted in Figure 2 are mean values from 6 aortic rings for each. Prazosin and cleistanthin B relaxed the precontracted aortic rings completely (Figure 2b) whereas cleistanthin A failed to do so in 
the available concentration range (Figure 2a). An inhibitory trend was seen with cleistanthin A but it was not statistically significant.The $\mathrm{IC}_{50}$ of cleistanthin A could not be calculated with the available results. Thus, it could not be compared with prazosin. The $\mathrm{pIC}_{50}$ of cleistanthin $\mathrm{B}$ was $5.721 \pm 0.6974$ (mean \pm standard deviation). The derived $\mathrm{IC}_{50}$ of cleistanthin B was approximately 10 times that of prazosin, but their $\mathrm{pIC}_{50}$ values were not statistically different (Table 1 ). The $\mathrm{IC}_{50}$ was derived from the mean $\mathrm{pIC}_{50}$ value. The relative inhibition produced by cleistanthin $\mathrm{A}$, cleistanthin $\mathrm{B}$ and prazosin relative to the vehicle control is demonstrated in Figure 2c.

\section{Prevention of phenylephrine-induced contractions}

The final concentration of the test compounds in the tissue bath was as follows, cleistanthin A (185 nM), cleistanthin B $(2.3 \mu \mathrm{M})$, prazosin $(65.2$ $\mathrm{nM})$ and vehicle control (50 $\mu \mathrm{l}$ of $10 \%$ ethanol). Prazosin demonstrated a characteristic parallel shift of the phenylephrine dose response curve to the right (Figure 3e). Cleistanthin A demonstrated a slight shift to the right and also led to the depression of the maximal response (Figure 3a). Concentrations higher than $185 \mathrm{nM}$ did not show any shift of the phenylephrine curves or reduction of the maximal response (Figure

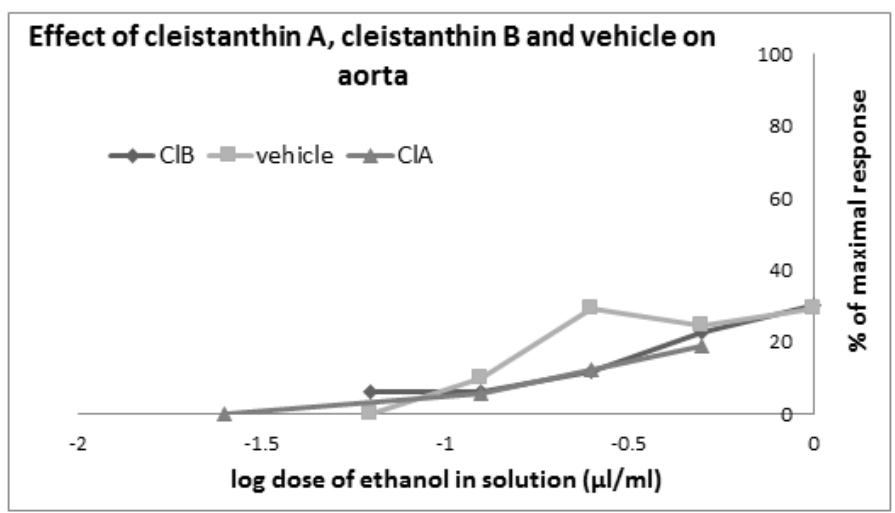

Figure 1: Dose response curve showing the agonistic response of cleistanthin $A$, cleistanthin $B$ and vehicle control on the isolated aortic rings of guinea pig when matched for their ethanol content.

$(\mathrm{N}=1$ for each) $\mathrm{Cl} A=$ Cleistanthin $\mathrm{A} ; \mathrm{ClB}=$ cleistanthin $\mathrm{B}$; vehicle = vehicle control

Table 1: Comparison of potency of cleistanthin B with prazosin in relaxing the phenylephrine precontracted aortic rings using $\mathbf{I C}_{50}$ values

\begin{tabular}{ccccc}
\hline S.No & Parameters & Prazosin & Cleistanthin B & Pvalue \\
\hline 1 & $\mathrm{pIC}_{50}($ mean $\pm \mathrm{SD})$ & $\begin{array}{c}6.752 \pm \\
1.064\end{array}$ & $5.721 \pm 0.6974$ & 0.0753 \\
2 & $\begin{array}{c}\text { Calculated } \mathrm{IC}_{50} \\
(\mathrm{M} / \mathrm{L})\end{array}$ & $1.77 \times 10^{-7}$ & $1.9 \times 10^{-6}$ & \\
3. & Potency ratio & 1 & 0.093 & \\
& & & \\
\end{tabular}

$\mathrm{pIC}_{50}$ is the negative logarithm of the $\mathrm{IC}_{50}$ values.

$\mathrm{pIC}_{50}$ values were obtained by log transforming the $\mathrm{IC}_{50}$ values for individual tissues $(\mathrm{N}=6)$ to achieve normal distribution. $\mathrm{pIC}_{50}$ was expressed as mean $\pm \mathrm{SD}$. The calculated $\mathrm{IC}_{50}$ is the antilog of mean $\mathrm{pIC}_{50}$. The $\mathrm{pIC}_{50}$ values were compared between the groups using the Student's t-test.

Potency ratio $=$ Calculated $\mathrm{IC}_{50}$ of prazosin $/$ Calculated $\mathrm{IC}_{50}$ of cleistanthin $\mathrm{B}$. It denotes that cleistanthin B is 0.093 times as potent as prazosin in inhibiting phenylephrine induced contractions in guinea pig aorta. 3b). Cleistanthin B strongly depressed the maximal response produced by phenylephrine at a working concentration of $2.3 \mu \mathrm{M}$ (Figure 3c). At a higher concentration of $23 \mu \mathrm{M}$, cleistanthin B almost completely suppressed any response with phenylephrine (Figure $3 \mathrm{~d}$ ). Though the maximal response was suppressed at $2.3 \mu \mathrm{M}$ dose of cleistanthin $\mathrm{B}$, there was an overlapping of curves and no discernible right shift. Also, there was a sustained response observed when cleistanthin B solution was added to the tissue bath during equilibration leading to a left shift of the initial part of the curves (Figure 3c). These aberrant responses were most likely caused by the interaction of vehicle ethanol with vascular tissue. This left shift was also seen in the preventive curves of vehicle control (Figure 3f).

\section{Inhibition of potassium chloride-induced contractions}

Neither cleistanthin A nor cleistanthin B had any effect on the potassium chloride-induced contractions (Figure $4 \mathrm{a}$ and $4 \mathrm{~b}$ ). In Figure $4 \mathrm{a}$, the effect of cleistanthin A on potassium chloride induced contractions and phenylephrine induced contractions were compared. Only the last dose (37 uM) showed a statistically significant difference $(p<0.05)$. The inhibitory effect of cleistanthin B was more profound on phenylephrine induced contractions and was absent on potassium chloride-induced contractions $(p<0.001)$. The relation is demonstrated in Figure $4 \mathrm{~b}$. Figure $4 c$ compares the effect of cleistanthin A, cleistanthin B and diltiazem on potassium chloride induced contractions. Diltiazem, a calcium channel blocker relaxed the tissue precontracted with potassium chloride whereas cleistanthins A and B had no effect.
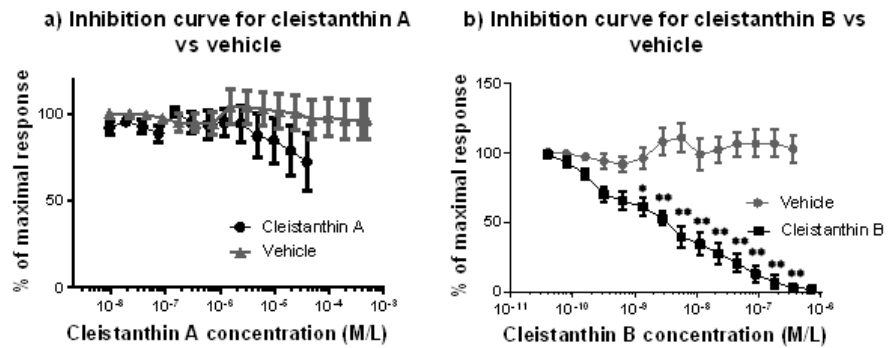

c) Comparis on of Cleistanthin A, Cleistanthin B and Prazosin

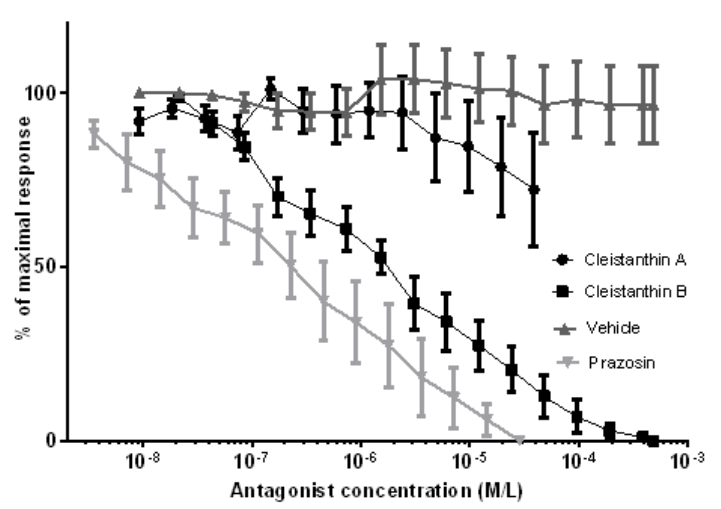

Figure 2: Dose response curves demonstrating the effect of test compounds on the phenylephrine induced contractions compared to vehicle control. The Figures demonstrate the effect of (a) cleistanthin A vs vehicle control $(n=6)$ and (b) cleistanthin B vs vehicle control $(n=6)$, and (c) comparison between cleistanthin A, cleistanthin B, prazosin and vehicle with doses in moles per liter on the phenylephrine induced contractions of curative protocol in guinea pig aortic rings. Two-way repeated measures ANOVA with bonferroni's post hoc was used for the comparison in Figures 2(a) and (b). Cleistanthin B produced significant reduction in response. ${ }^{*}$ indicates $p<0.05$ and ${ }^{* *}$ indicates $p<0.001 . \mathrm{ClA}=$ cleistanthin $\mathrm{A} ; \mathrm{ClB}=$ cleistanthin $\mathrm{B} ;$ Vehicle = vehicle control. 


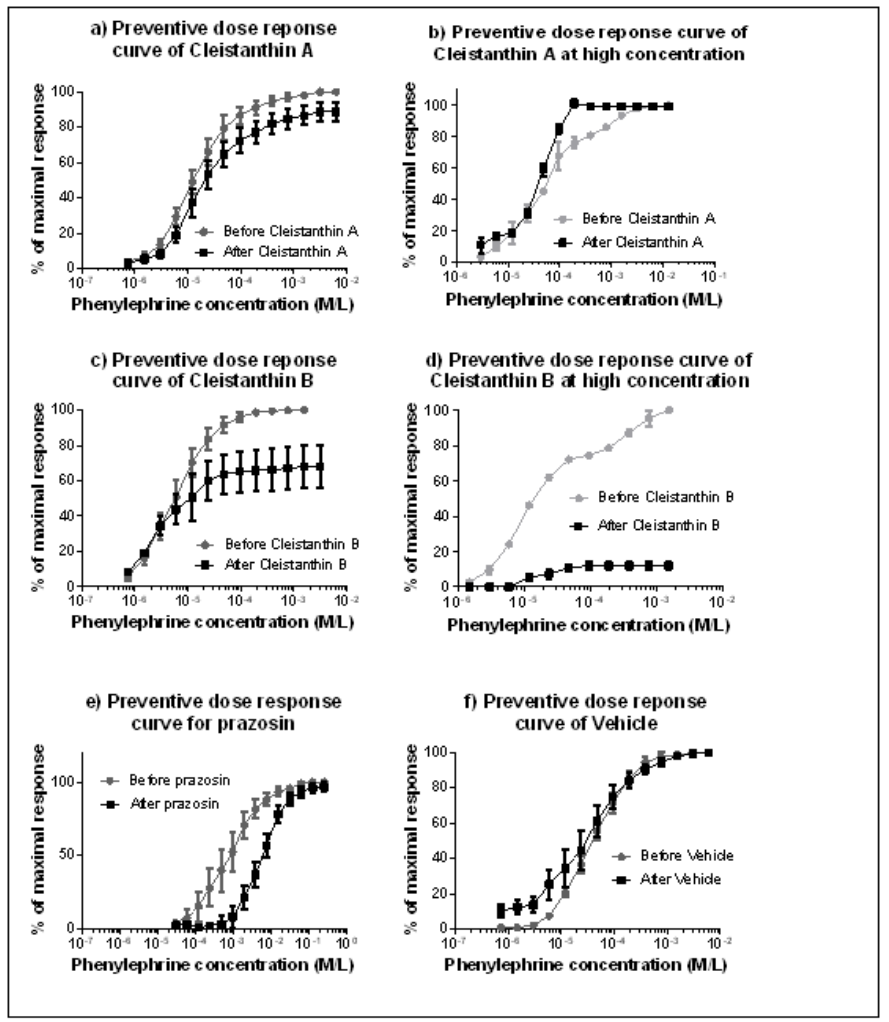

Figure 3: Preventive dose response curves demonstrating the effect of (a) low dose cleistanthin $A(N=6)$, (b) high dose cleistanthin $A(N=2)$, (c) low dose cleistanthin $B(N=6)$, (d) high dose cleistanthin $B(N=2)$, (e) prazosin $(N=6)$ and (f) vehicle control $(\mathrm{N}=6)$ on the phenylephrine dose response curves.

\section{DISCUSSION}

In the protocol where the relaxation of aortic tissues precontracted with phenylephrine was tested, cleistanthin B fully relaxed the tissues whereas cleistanthin A failed to do so. However, most of the tissues treated with cleistanthin A displayed an initial dip in the curve followed by an increase in contraction (Figure S1). Prazosin is a highly potent $\alpha_{1}$ adrenergic blocker and cleistanthin B potency ratio is 0.093 times (based on derived $\mathrm{IC}_{50}$ values in Table 1) that of prazosin making it a very potent $\alpha_{1}$ blocker but the difference between the $\mathrm{pIC}_{50}$ values was not statistically significant (Table 1). The potency of cleistanthin A could not be demonstrated as it did not reach $50 \%$ inhibition. The guinea pig aortic rings primarily contain $\alpha_{1}$ adrenoceptors postsynaptically. ${ }^{30}$ Thus, the ability of cleistanthin $B$ to relax the aortic rings pre-contracted with phenylephrine points to $\alpha_{1}$ adrenoceptor inhibition. This inhibition in guinea pig aortic tissues has been demonstrated with Cleistanthus collinus leaf extract. ${ }^{28}$ The hypotensive response of both compounds has been demonstrated in vivo in rats. ${ }^{18,19}$ Also, in vitro studies in guinea pig vas deferens with cleistanthins $\mathrm{A}$ and $\mathrm{B}$ provide additional evidence towards $\alpha_{1}$ adrenergic receptor blockade. ${ }^{24}$ Another study from our lab has also demonstrated that cleistanthins A and B inhibit both $\alpha_{1}$ adrenergic receptors (in jejunum) and $\alpha_{2}$ adrenergic receptors (in platelets) in rats. ${ }^{31}$ But in our experiment, cleistanthin A did not demonstrate a significant inhibition in the dose ranges used. The reason for this might be the fact that the lower concentration of cleistanthin A in the solution and higher concentration of ethanol might have interfered in the inhibition process leading to an altered response. As reported in some previous studies, ethanol is not inert and increases the sensitivity of aortic tissue to phenylephrine..$^{32,33}$ The same was corroborated in our
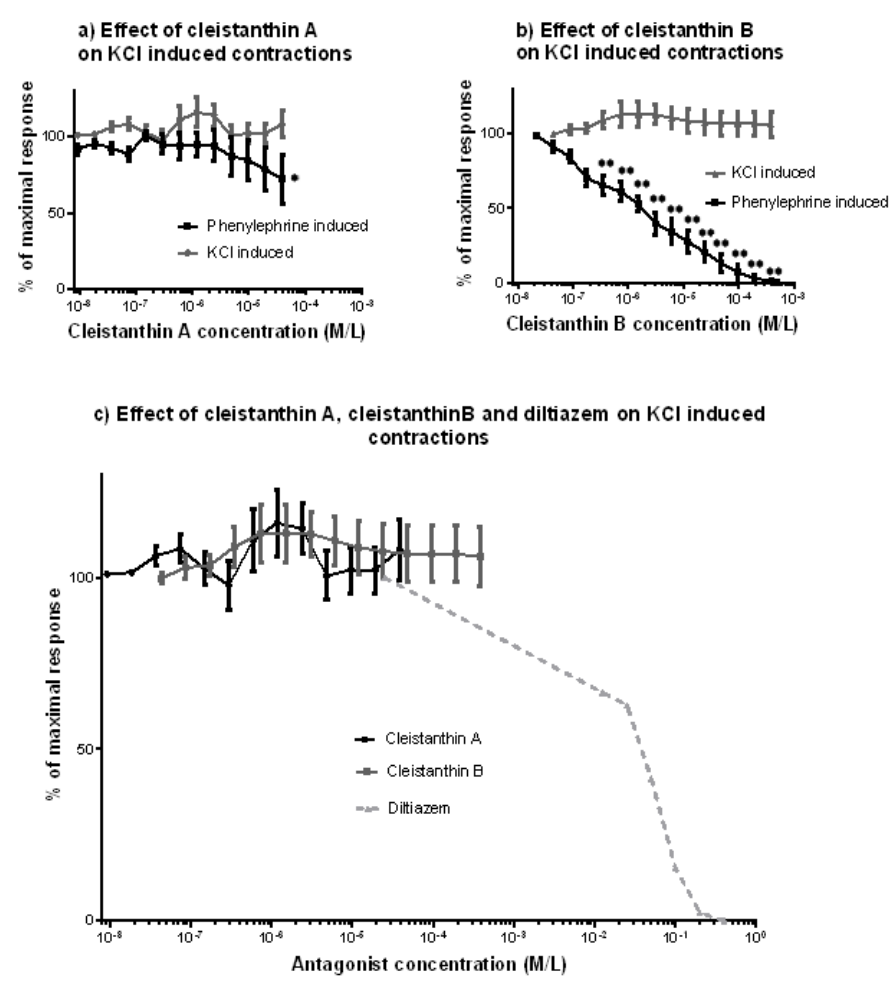

Figure 4: Inhibitory curves demonstrating the effect of (a) cleistanthin A and (b)cleistanthin $\mathrm{B}$ on the $\mathrm{KCl}$ induced contractions compared to its effect on the phenylephrine induced contractions and (c) cleistanthin A and cleistanthin $\mathrm{B}$ against diltiazem on $\mathrm{KCl}$ induced contractions. ( $\mathrm{N}=6$ for cleistanthin $\mathrm{A}$ and $\mathrm{B}$ but $\mathrm{N}=1$ for diltiazem).

Two-way repeated measures ANOVA with Bonferroni's post hoc was used for the comparison in Figures $4(a)$ and $(b){ }^{*}$ indicates $p<0.05$ and ${ }^{* *}$ indicates $p<0.001 . \mathrm{Cl} A=$ cleistanthin $\mathrm{A} ; \mathrm{ClB}=$ cleistanthin $\mathrm{B} ;$ Vehicle $=$ vehicle control
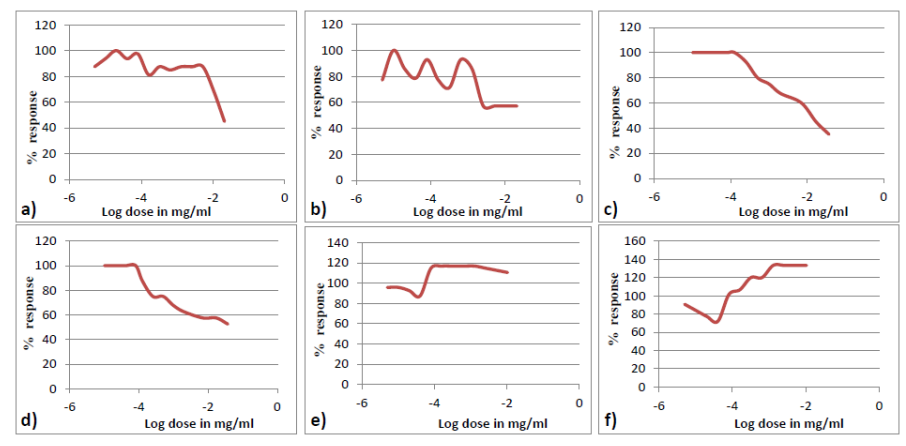

Supplement 1: Effect of cleistanthin A on phenylephrine induced contractions in each of the 6 guinea pig aortic rings (a-f).

The concentration of cleistanthin A added in the tissue bath was expressed in $\mathrm{mg} / \mathrm{ml}$ and converted to log dose and plotted against percentage response. Bath volume $=40 \mathrm{ml}$.

study as cleistanthin A, cleistanthin B and vehicle control independently contracted the guinea pig aortic rings due to the solvent (Figures 1 and Figure 3f). It is worth noting that when the doses of the three were matched based on the ethanol content of the added solution; the responses were similar (Figure 1) and easily reversed by prazosin. This finding is in contrast to studies of cleistanthins A and B on guinea pig vas deferens where they did not report such a response with ethanol as the solvent. ${ }^{24}$ This is probably because aortic tissues are more sensitive to ethanol than vas deferens. This suggests that the contractions are due to 
the ethanol in which the two compounds are dissolved. The cleistanthin A treated precontracted tissues did show an initial dip in the curves which was abolished at higher concentration. This dip coincides with the inhibition curve of cleistanthin B when plotted together (Figure 2c) and is not seen in potassium chloride-induced contractions. So, it can be speculated that cleistanthin A does relax the tissues precontracted with phenylephrine like cleistanthin B with similar potency but due to its poorer solubility, the higher concentration of ethanol in the solvent interferes with the inhibition.

In the protocol involving the prevention of phenylephrine contraction, cleistanthin A demonstrated a adrenergic blockade by shifting the phenylephrine dose response curve to the right and suppressing the maximal response at a concentration of $185 \mathrm{nM}$. The depression of maximal response was also seen with cleistanthin B at $2.3 \mu \mathrm{M}$. This suggests that both are noncompetitive blockers of $\alpha_{1}$ adrenergic receptors. This explains why Cleistanthus collinus poisoning causes hypotension resistance to inotropes as reported in some case reports. ${ }^{3}$ But like the curative method, the response with cleistanthin A was inconsistent due to the interaction of the solvent ethanol. At higher concentration, i.e. $1.85 \mu \mathrm{M}$ it failed to display any right shift or suppression of maximal response with a slight shift to the left (Figure 3b). Concentrations higher than this could not be achieved due to poor solubility of cleistanthin A. A similar leftward shift of the phenylephrine curve after adding vehicle control (ethanol $50 \mu \mathrm{l}$ of $10 \%$ ethanol) provides evidence of this interaction produced by ethanol. The ethanol affected the phenylephrine prevention curves of cleistanthin $\mathrm{B}(2.3 \mu \mathrm{M})$ too, as the characteristic shift to the right was distorted due to crossing of the curves (Figure $4 c$ ) but the inhibition of maximal response was prominent. In previous studies of cleistanthins A and B on guinea pig vas deferens, a right shift of the phenylephrine curve was observed. ${ }^{24}$ Because of the higher solubility of cleistanthin B a higher concentration could be achieved and at $23 \mu \mathrm{M}$ concentration, it reduced the maximal response to $20 \%$ of its original demonstrating a consistent dose-dependent non-competitive response. In contrast to cleistanthins $\mathrm{A}$ and $\mathrm{B}$, prazosin demonstrated a classical competitive inhibitory curve with a parallel shift to right and sustained maximal response (Figure $3 \mathrm{e}$ ). The calculation of the antagonist equilibrium constants $\left(\mathrm{K}_{\mathrm{B}}\right)$ was not attempted as the separation of the preventive curves was minimal owing to the alteration of the curves by ethanol. Any attempt at the derivation of $K_{B}$ from the curves would give erroneous results. The effect of cleistanthins $\mathrm{A}$ and $\mathrm{B}$ were also studied on potassium chloride- induced contractions to exclude the role of calcium channel blockade in the inhibition produced. Calcium channel blockers have been shown to inhibit potassium chloride induced contractions. ${ }^{25}$ This fact was also demonstrated by using diltiazem in one aortic ring (Figure 4c). Both cleistanthin A and cleistanthin B failed to relax the tissue pre-contracted with potassium chloride at the doses that produced relaxation in phenylephrine contracted tissues (Figure $4 \mathrm{a}$ and b). In the case of cleistanthin B, the lack of response was obvious. However, the difference between the effect of cleistanthin A on potassium chloride and phenylephrine-induced contractions was statistically different only at the last dose. This is because ethanol affects the phenylephrine induced contractions and not the potassium chloride-induced contractions. ${ }^{32}$ Thus, the inhibition of phenylephrine induced contractions was delayed till a higher concentration of cleistanthin A was achieved. These findings suggest that cleistanthins $A$ and $B$ act by irreversibly inhibiting $\alpha_{1}$ adrenergic receptors and not by blocking voltage-gated calcium channels. The choice of ethanol as the solvent was a major limitation of the study. It interfered with all the responses leading to altered responses with both cleistanthins A and B. Ethanol was chosen because cleistanthin A and $\mathrm{B}$ were not soluble in most common solvents and in previous studies ethanol was used as the solvent. ${ }^{24}$
Considering the interest in the anticancer potential of cleistanthins A and $\mathrm{B}$ and their derivatives, this data reveals an important off-target effect that needs to be addressed. Also, this vasodilatory non-competitive $\alpha_{1}$ adrenergic property may prove useful in the treatment of conditions like severe hypertension and benign prostatic hyperplasia (provided similar action is demonstrated in the urinary tract). More importantly, this finding helps us understand the mechanism behind the hypotension in Cleistanthus collinus poisoning which has been associated with increased mortality. ${ }^{1}$ This might aid us in our search for an effective antidote for Cleistanthus collinus poisoning. To explore the therapeutic and toxic potential of these compounds, we need a detailed understanding of the actions of cleistanthins A and B and their derivative compounds.

\section{CONCLUSION}

Both cleistanthins A and B have vasodilatory action on guinea pig aorta. The action is through $\alpha_{1}$ adrenergic receptor inhibition and calcium channel blockade does not contribute to the inhibition. They noncompetitively inhibit the $\alpha_{1}$ adrenergic receptors in guinea pig aorta. The inhibition produced by cleistanthin B is comparable to prazosin.

\section{Research funding}

The study was funded by the Institutional Intramural grant for postgraduate research in JIPMER.

\section{CONFLICT OF INTEREST}

The authors state no potential conflict of interest.

\section{Ethical approval}

The research related to animals' use has complied with all the relevant national regulations and institutional policies for the care and use of animals.

\section{ABBREVIATIONS}

EC $_{50}$ : Effective phenylephrine concentration eliciting $50 \%$ of the maximal response; $\mathbf{I C}_{50}$ : Antagonist concentration inhibiting the maximal response by $50 \%$; $\mathbf{K}_{\mathrm{B}}$ : Antagonist equilibrium constants; $\mathrm{KCl}$ : Potassium chloride; $\mathrm{pIC}_{50}$ : Negative logarithm of $\mathrm{IC}_{50} ;$ PSS: Physiological salt solution.

\section{REFERENCES}

1. Subrahmanyam DKS, Mooney T, Raveendran R, Zachariah B. A clinical and laboratory profile of Cleistanthus collinus poisoning. J Assoc Physicians India. 2003;51:1052-4.

2. Eswarappa S, Chakraborty AR, Palatty BU, Vasnaik M. Cleistanthus collinus poisoning: Case reports and review of the literature. J Toxicol Clin Toxicol. 2003;41(4):369-72.

3. Benjamin SPE, Fernando ME, Jayanth JJ, Preetha B. Cleistanthus collinus poisoning. J Assoc Physicians India. 2006;54:742-4.

4. Das S, Hamide A, Mohanty MK, Muthusamy R. Fatal Cleistanthus collinus toxicity: A case report and review of the literature. J Forensic Sci. 2014;59(5):1441-7.

5. Shankar V, Jose VM, Bangdiwala SI, Thomas K. Epidemiology of Cleistanthus collinus (oduvan) poisoning: Clinical features and risk factors for mortality. Int J Inj Contr Saf Promot. 2009;16(4):223-30.

6. Bammigatti C, Surynarayana BS, Kumar HKT, Kumar GS. Pattern and outcome of Cleistanthus collinus (Oduvanthalai) poisoning in a tertiary care teaching hospital in South India. J Forensic Leg Med. 2013;20(8):959-61.

7. Chrispal A. Cleistanthus collinus poisoning. J Emerg Trauma Shock. 2012;5(2):160-6.

8. Raveendran R, Parasuraman S, Gopal V. Isolation and purification of cleistanthin $\mathrm{A}$ and $\mathrm{B}$ from the leaves of Cleistanthus collinus Rob. (Euphorbiaceae) Arch Pharm Sci Res. 2009;1:199-202.

9. Anjaneyulu ASR, Ramaiah PA, Row LR, Venkateswarlu R, Pelter A, Ward RS. New lignans from the heartwood of Cleistanthus collinus. Tetrahedron. 1981;37(21):3641-52.

10. Annapoorani KS, Periakali P, llangovan S, Damodaran C, Sekharan PC. 
Spectrofluorometric determination of the toxic constituents of Cleistanthus collinus. J Anal Toxicol. 1984;8(4):182-6.

11. Govindachari TR, Sathe SS, Viswanathan N, Pai BR, Srinivasan M. Chemical constituents of Cleistanthus collinus (Roxb.). Tetrahedron. 1969;25(14):2815-21.

12. Subramanian R, Krishnamurthy G. Thin-layer chromatographic detection of the lignan lactones of Cleistanthus collinus (Roxb.). J Chromatogr. 1975 9;107(1):230-3.

13. Pradheepkumar CP, Shanmugam G. Anticancer potential of cleistanthin A isolated from the tropical plant Cleistanthus collinus. Oncol Res. 1999;11(5):22532.

14. Suman T, Elangomathavan R, Soundherrajan I, Ramesh S. In vitro cytotoxic effect of Cleistanthus collinus extracts and fractions on mouse cell line. J App Pharm Sci. 2014;4(10):44-6.

15. Thummar VR, Parasuraman S, Basu D, Raveendran R. Evaluation of in vivo antitumor activity of cleistanthin B in Swiss albino mice. J Tradit Complement Med. 2015;6(4):383-8

16. Elangomathavan R, Suman T, Beaulah SN, Ramesh S, Patharajan S. Comparative analysis of Cleistanthus collinus aqueous leaf extract and fractions for its antibacterial potential. Int J Enteric Pathog. 2015;3(2):e22946.

17. Kovendan K, Arivoli S, Maheshwaran R, Baskar K, Vincent S. Larvicidal efficacy of Sphaeranthus indicus, Cleistanthus collinus and Murraya koenigii leaf extracts against filarial vector, Culex quinquefasciatus Say (Diptera: Culicidae). Parasitol Res. 2012;111(3):1025-35.

18. Priyadharsini RP, Parasuraman $S$, Raveendran R. Evaluation of the antihypertensive activity and alpha-adrenergic receptor interaction of cleistanthins A and B. J Basic Clin Pharm. 2014;5(4):109-14.

19. Parasuraman $S$, Raveendran R, Selvaraj RJ. Effects of cleistanthins A and B on blood pressure and electrocardiogram in Wistar rats. Z Für Naturforschung C J Biosci. 2011;66(11-12):581-7.

20. Parasuraman S, Raveendran R. The effects of aqueous extract of Cleistanthus collinus (Roxb.) (Euphorbiaceae) leaves on rat blood pressure. Pharmacogn Res. 2012;4(3):178-80.

21. Kettimuthu KP Lourthuraj AA, Manickam AS, Subramani S, Ramachandran A. Mechanisms of toxicity of Cleistanthus collinus: Vacuolar ATPases are a putative target. Clin Toxicol Phila Pa. 2011;49(6):457-63.

22. Zhang Z, Ma J, Zhu L, Zhao Y. Synthesis and identification of cytotoxic diphyllin glycosides as vacuolar H(+)-ATPase inhibitors. Eur J Med Chem. 2014;82:46671.
23. Shen W, Zou X, Chen M, Liu P, Shen Y, Huang S, et al. Effects of diphyllin as a novel V-ATPase inhibitor on gastric adenocarcinoma. Eur J Pharmacol. $201 ; 667(1-3): 330-8$

24. Parasuraman $S$, Raveendran R. Effect of cleistanthin $A$ and $B$ on adrenergic and cholinergic receptors. Pharmacogn Mag. 2011;7(27):243-7.

25. Marriott JF. A comparison of the effects of the calcium entry blockers, verapamil, diltiazem and flunarizine against contractions of the rat isolated aorta and portal vein. Br J Pharmacol. 1988;95(1):145-54.

26. Mengistu $M$, Abebe $Y$, Mekonnen $Y$, Tolessa T. In vivo and in vitro hypotensive effect of aqueous extract of Moringa stenopetala. Afr Health Sci. 2012;12(4):54551.

27. Ghayur MN, Gilani AH. Ginger lowers blood pressure through blockade of voltage-dependent calcium channels. J Cardiovasc Pharmacol. 2005;45(1):7480 .

28. Kumar MR, Ramaswamy S, Jayanthi M, Raveendran R. Alpha-adrenergic receptor blocking effect of Cleistanthus collinus (Roxb.) Benth. and Hook f. leaf extract on guinea pig isolated smooth muscle preparations. Indian J Exp Biol. 2011;49(5):339-42.

29. Parasuraman S, Raveendran R, Madhavrao C. GC-MS analysis of leaf extracts of Cleistanthus collinus Roxb. (Euphorbiaceae). Int J Ph Sci. 2009;1(2):284-6.

30. Ruffolo RR, Waddell JE, Yaden EL. Heterogeneity of postsynaptic alphaadrenergic receptors in mammalian aortas. J Pharmacol Exp Ther 1982;221(2):309-14.

31. Lakshmanan M, Zachariah B, Raveendran R. Action of cleistanthins A and B on alpha adrenoceptors in rats. J Young Pharm. 2016;8(3):177-85

32. Masui H, Wakabayashi I, Hatake K, Yoshimoto S, Nakamura $Y$, Seto $Y$, et al. Potentiating effect of ethanol on alpha 1 adrenergic receptor mediated contraction of thoracic aorta from guinea pig. Nihon Arukōru Yakubutsu Igakkai Zasshi Jpn J Alcohol Stud Drug Depend. 1997;32(2):107-21.

33. Masui H, Wakabayashi I, Hatake K, Kurahashi M, Yoshimoto S, Hishida S, et al. Effect of ethanol on isometric tone of aorta from guinea pig1. Arukōru Kenkyū Yakubutsu Izon Jpn J Alcohol Stud Drug Depend. 1993;28(3):79-94.

Article History: Submission Date : 20-02-2021; Revised Date : 21-03-2021; Acceptance Date : 03-06-2021.

Cite this article: Sahoo FK, Girish C, Raveendran R. Effect of Cleistanthins A and B on the Isolated Aorta of Guinea Pig. J Young Pharm. 2021;13(2):107-112. 